\title{
STEPPS (System Training of Emotional Predictability and Problem Solving) in einer vernetzten ambulanten Hilfe für Menschen mit Borderline Persönlichkeitsstörung
}

\author{
Ewald Rahn
}

Online publiziert: 30. November 2020

(c) Der/die Autor(en) 2020

\begin{abstract}
Zusammenfassung STEPPS ist ein evidenzbasiertes Fertigkeitentraining für Menschen mit einer Borderline Persönlichkeitsstörung, das sich vor allem für eine langfristige ambulante Begleitung eignet. STEPPS ist ein Gruppenprogramm und dient als Ergänzung zu bereits bestehenden Hilfeformen, weil eine optimale Nutzung der vorhandenen Hilfen ein Ziel des Trainings ist. Das Programm umfasst eine Auseinandersetzung mit der Erkrankung, die Vermittlung von Fertigkeiten zum Umgang mit Emotionen und ein Verhaltenstraining zum Transfer der vermittelten Fertigkeiten. Das soziale Netz der Betroffenen wird einbezogen und eine nachhaltige Partizipation angestrebt. Damit dient das Programm auch der indizierten Prävention der ausgeprägten psychosozialen und gesundheitlichen Risiken der Erkrankung. Das Programm ist besonders für vernetzte Versorgungssysteme geeignet.
\end{abstract}

Schlüsselwörter Borderline Persönlichkeitsstörung · Langfristiges ambulantes Therapieprogramm . Trialogisch • Einbezug des sozialen Netzwerkes . Vereinbarkeit mit anderen Hilfen Prävention langfristiger sozialer und gesundheitlicher Folgen . Förderung der Selbsthilfe

\section{STEPPS (System Training of Emotional Predictability and Problem Solving) in a networked outpatient assistance for people with a Borderline Personality disorder}

Summary STEPPS is an evidence-based skill training for people with borderline personality disorder, which is especially suitable for long-term outpatient support. STEPPS is a group program and serves as

\section{E. Rahn $(\bowtie)$}

LWL Kliniken im Kreis Soest, Warstein, Deutschland info@rahn76.de a supplement to already existing forms of help, because optimal use of the available help is a goal of the training. The program includes an examination of the disease, the teaching of skills to deal with emotions and behavioral training for the transfer of the skills taught. The social network of those affected will be involved and sustainable participation will be sought. Thus, the program also serves the indexed prevention of the pronounced psychosocial and health risks of the disease. The program is particularly suitable for networked supply systems.

Keywords Borderline personality disorder · Longterm outpatient therapy program - Trialogical · Inclusion of the social network - Compatibility with other aids Prevention of long-term social and health consequences · Promoting self-help

\section{Einführung}

Die Borderline Persönlichkeitsstörung (BPD) zeichnet sich durch emotionale Instabilität, Anfälligkeit für Stress und Störungen der sozialen Interaktion aus. Die Störung hat erhebliche Auswirkungen auf das Selbstbild (instabiles, negatives Selbstbild, innere Leere, Entfremdungserleben), die Selbststeuerung (Zielsetzungen, Interessen, Wertvorstellungen, berufliche Pläne) und die zwischenmenschlichen Beziehungen (Mentalisierungsschwäche, Missinterpretationen und Erleben sozialer Ablehnung in neutralen Situationen, Misstrauen und Schwierigkeiten, die Kooperation nach empfundenen Kränkungen fortzuführen). Die Störung der zwischenmenschlichen Beziehungen betreffen enge Beziehungen (ängstliche Beschäftigung mit tatsächlichem oder vermeintlichem Verlassenwerden, Wechsel von Anhänglichkeit und Ablehnung) aber auch weniger enge Beziehungen (MitschülerInnen, ArbeitskollegInnen etc.) (Keuroghlian et al. 
2013). Nicht zuletzt sind auch die Beziehungen zu den HelferInnen von den Störungen der sozialen Interaktionen betroffen (Aktive Einforderung von Hilfe bei Empfindlichkeit gegenüber Kränkungen und Kritik, Misstrauen, Schwierigkeiten eigene Ressourcen zu nutzen).

Besonders zu Beginn der Erkrankung treten eine Reihe von Begleitphänomenen auf, die oft mit der Stressanfälligkeit der Betroffenen zusammenhängen, etwa chronische Suizidalität, Selbstverletzungen und Substanzmissbrauch. Die damit oft verbundenen krisenhaften Zuspitzungen führen zu häufigen Aktivierungen der Notfallsysteme, vor allem zu Kriseninterventionen in psychiatrischen Krankenhäusern (Wagner et al. 2013). Die stationären Behandlungen und die damit assoziierten Störungen der psychosozialen Entwicklung, insbesondere eine fehlende berufliche Integration, erzeugen die hohen Krankheitskosten zu Beginn der Erkrankung (Zanarini et al. 2010).

Die BPD ist vor allem eine Krankheit von Adoleszenten und jungen Erwachsenen mit einer hohen Prävalenz $(-5 \%)$. Langzeitstudien zeigen allerdings eine hohe Remissionsrate zwischen 50 und $88 \%$ im weiteren Verlauf (Zanarini et al. 2006; Biskin 2015). Viel weniger positiv entwickeln sich die psychosozialen Funktionen der Betroffenen und die Partizipation. Bereits zu Beginn zeigen die Betroffenen Teilhabeeinschränkungen, etwa in Schule und Beruf, der Einbindung in die Peer Group, der Güte der familiären Bindungen und in intimen Beziehungen (Stone 2016). Häufige Notfallaufnahmen in psychiatrischen Krankenhäusern mit oft langen stationären Behandlungen verstärken diesen Trend. Dadurch steigt die Gefahr einer multifunktionalen Abhängigkeit von psychosozialen Hilfen. Möglich ist, dass mit der Zeit die Betroffenen ihr gesamtes soziales Netz aus professionellen HelferInnen rekrutieren. Die Einschränkungen bei den psychosozialen Funktionen prägen damit den langfristigen und chronischen Verlauf der BPD. (Lis, S., \& Bohus, M. 2013). Sie bilden sich nur langsam und teilweise zurück und führen zu weiteren Folgestörungen, wie sozialer Isolation, einer Häufung schwerwiegender körperlichen Erkrankungen (Frankenburg, F.R., \& Zanarini, M.C. 2005) und Abhängigkeit von psychosozialen Hilfen. Besonders problematisch ist die berufliche Integration (Zanarini et al. 2010; Kramer et al. 2017; Álvarez-Tomás et al. 2018). Für die Entwicklung der psychosozialen Funktionseinschränkungen ist offensichtlich nicht nur die Störung selbst, sondern auch die prämorbide Entwicklung beteiligt, etwa durch Ausbildung maladaptiver Schemata, Folgen komplexer Traumatisierung und ungünstiger psychosozialer Entwicklungsbedingungen (Bozzatello et al. 2019).
Welche besonderen Herausforderungen gibt es in der Behandlung der BPD?

In den letzten 20 Jahren sind durch die Einführung evidenzbasierter Therapieverfahren deutliche Verbesserungen in der Behandlung der BPD erzielt worden. Alle Verfahren sind multimodal aufgebaut, in der Regel manualisiert und trennen zwischen (möglichst kurzer) Krisenintervention und langfristigen entwicklungsorientierten Hilfen. In allen Verfahren ist die Rolle des/r TherapeutIn (HelferIn) beschrieben. Der größte Teil der Verfahren ist für ein ambulantes oder tagesklinisches Setting entwickelt, können aber mehr oder weniger auf stationäre Settings angepasst werden. Abgesehen von einer globalen Wirksamkeit unterscheiden sich die Verfahren in Hinblick auf die Effekte auf spezifische Wirkfelder (Stresstoleranz, Gefährdung, Krisenanfälligkeit, zwischenmenschliche Beziehungen, Selbstregulation, Autonomie u. a.). Die Beeinflussung der psychosozialen Funktionen hat nicht in allen Verfahren einen Platz und ist deswegen auch noch wenig untersucht.

Im gegenwärtigen psychiatrisch/psychotherapeutischen Versorgungssystem lassen sich jedoch multimodale Verfahren vor allen bei den ambulanten Hilfen nur unvollständig integrieren, weil die dafür notwendige Vernetzung von ambulanten, teilstationären und stationären Angeboten sich oft schwierig gestaltet. Die Hilfen für die Betroffenen sind daher oft unvollständig, unzureichend integriert, bilden den jeweiligen Entwicklungsstand nicht ab und erfolgen nicht zeitnah genug. Im Hinblick auf die längerfristige Entwicklung fehlen zudem noch Konzepte im Hinblick auf die soziale und berufliche Teilhabe.

Aus den bisherigen Erfahrungen lassen sich daher Anforderungen herleiten, denen zukünftige Entwicklung der Behandlung Borderline Kranker Rechnung tragen sollten

1. Die Angebote sollten den spezifischen Aspekten der Erkrankung Rechnung tragen,

2. eine Vernetzung unterschiedlicher Versorgungssegmente ermöglichen,

3. für die Betroffenen und deren Angehörige zugänglich sein,

4. eine angemessene Haltung gegenüber den Betroffenen vermitteln,

5. auf die Reduktion der Symptome aber auch auf die Verbesserung der Partizipation (soziale und berufliche Integration) zielen,

6. die Pflege und den Erhalt der sozialen Netzwerke ermöglichen,

7. mit den allgemeinen Rahmenbedingungen in der Versorgungsrealität vereinbar sein

8. und der Vermeidung häufiger stationärer Krisenintervention dienen.

Alle bisher entwickelten Behandlungsverfahren können diesen Anforderungen nur zum Teil Rechnung tragen. Das gilt auch für das STEPPS Programm. Zu- 
Tab. 1 Die Themen des Basisprogramms

\begin{tabular}{|l|l|l|}
\hline 1. Schritt & 2. Schritt & 3. Schritt \\
\hline Einführung in die Gruppe & Distanzieren & Ziele setzen \\
\hline Vereinbarungen & Kommunizieren & Essen \\
\hline Die Krankheit erkennen und annehmen & Gedanken hinterfragen & Schlafen \\
\hline Die Instrumente von STEPPS & Sich ablenken & Bewegung \\
\hline Schema & Probleme bewältigen & Freizeit gestalten \\
\hline & & Körperliche Gesundheit \\
\hline & & Vermeidung von Missbrauch und Selbstschädigung \\
\hline
\end{tabular}

\section{Tab. 2 STAIRWAYS Themen}

\section{1. +2. Sitzung \\ Ziele setzen \\ 1. problematische \\ Bereiche identifizieren \\ 2. sich ein Ziel setzen \\ 3. Unterziele festlegen \\ 4. sich die erfolgreiche Umsetzung \\ des Ziels vorstellen \\ 5. Aufschübe vermeiden \\ 6 . Fortschritte einschätzen und das \\ Ziel anpassen}

\section{1.-15. Sitzung}

Zwischenmenschliches Verhalten

Vertrauen.

Konflikte. Regeln für einen fairen

Streit.

Die eigenen Strategien und die anderen Personen beobachten.

Verzeihen und die Bedeutung von

Verzeihen für den eigenen Gesun-

dungsprozess.

Alles-oder-Nichts-Denken verändern

\section{3. +4. Sitzung}

Neues ausprobieren

Was passiert, wenn ich Neues ausprobiere?

Vor- und Nachteile abwägen. Ideen entwickeln der eigene Fortschritt wird im Verlauf des Trainings immer wieder gemeinsam überprüft

\section{6. + 17. Sitzung}

Vorbereitung auf schwierige Situationen

Erkennen, welche Situationen emotionale Krisen auslösen. Schutzstrategien im Vorhinein entwickeln und anwenden. Ein Skript/Drehbuch für die Situationen entwerfen

\section{5.-7. Sitzung}

Umgang mit Wut

Wut ein natürliches Gefühl.

Wut und Kontrolle.

Gesundheitliche Folgen unterdrückter

Wut.

Mit Wut so umgehen, dass man sich und anderen nicht schadet.

Situationen erkennen die Wut auslösen. Distanzieren.

Über Wut sprechen

Wut hinterfragen.

Krisenmanagement

\section{8.-20. Sitzung}

Selbstbehauptungstraining

Behauptende Verhaltensweisen erarbeiten.

Für die eigenen Rechte einstehen ohne die anderer zu verletzen

Nein sagen lernen,

mit den nein eines anderen umgehen lernen

\section{8.-10. Sitzung}

Impulskontrolle

Gewohnheiten, die einem selbst schaden erkennen.

Sich Ausrutscher und Rückfälle verzeihen und als Signale verstehen. Auslöser erkennen. Ablenkungstechniken erweitern. Aktiv handeln, nicht reagieren. Eigene Stärken und Kraftreserven erkennen

\section{1. + 22. Sitzung}

Sich zwischen Alternativen entscheiden

Gesunde Entscheidungen treffen, sich gegen ungesunde Entscheidungen entscheiden.

Beispiele betrachten, in denen Entscheidungen gut gelungen sind

\section{3. + 24. Sitzung}

\section{Am Ball bleiben}

\section{Recovery/Gesundung.}

Wie gelingt es im Leben weiterhin die Balance zu halten.

Wissen um ungesunde Beziehungsstrukturen einsetzen und sich für Alternativen entscheiden.

Sich erlauben, sich gut um sich selbst zu kümmern.

Um Hilfe bitten, wenn es nötig ist

künftig erscheint daher eine Methodenintegration (Herpertz 2020) sinnvoll, aber auch die Entwicklung neuer Elemente in der Versorgung, etwa Methoden zur Prävention oder indizierten Rehabilitation. Ein Fokus des STEPPS Programms liegt auf der Aktivierung sozialer Ressourcen zur Bewältigung der Störung und verfolgt einen trialogischen Ansatz. Es kann daher als Beispiel eines vorwiegend ambulanten und längerfristig angelegten Angebotes zur Behandlung der BPD dienen.

\section{Was ist das STEPPS Programm?}

STEPPS (System Training for Emotional Predictability and Problem Solving) ist ein manualisiertes Fertigkeiten Training (Skillstraining) für Menschen mit einer
Borderline-Persönlichkeitsstörung. Das Programm wurde von einer Arbeitsgruppe um Nancy Blum in Iowa entwickelt und erstmals im Jahre 2002 veröffentlicht. Im Jahr 2012 erfolgte eine Überarbeitung des Programms (Blum et al. 2012). STEPPS (Tab. 1) ist ein manualisiertes Gruppenprogramm mit 20 Sitzungen mit einer Dauer von 2h. Später ergänzten die Autoren das Basisprogramm durch STAIRWAYS (Tab. 2), ebenfalls ein Gruppenprogramm, mit insgesamt 24 Sitzungen verteilt über ein Jahr (Blum \& John, S.D. 2008).

STEPPS ist evidenzbasiert mit insgesamt drei randomisierten Kontrollstudien (RCTs) (Blum et al. 2008; van Wel et al. 2009; Bos et al. 2010; Bos et al. 2011; Schuppert et al. 2009). Das Programm modifiziert Stimmung und Impulsivität positiv, reduziert die 
Borderline typischen Symptome wie emotionale Instabilität, negative Kognitionen, erhöht die Beziehungsfähigkeit und verbessert das Selbstbild. STEPPS reduziert außerdem die Rehospitalisierungsrate. Ein Vergleich mit der dialektisch Behaviorale Therapie (DBT) erbrachte eine merkliche Reduktion von Symptomen, wobei durch die DBT akute Suizidalität und Selbstverletzungen deutlicher reduziert werden konnte (Guillén Botella et al. 2020). STEPPS beeinflusst dafür deutlicher die psychosoziale Funktionsfähigkeit der TeilnehmerInnen.

STEPPS wird mittlerweile nicht nur in den USA angewendet, sondern auch in mehreren europäischen Ländern (Deutschland, Niederlande, Großbritannien und Italien). In Deutschland wurde STEPPS 2014 eingeführt, zunächst als Nachsorgeprogramm für zuvor stationär behandelte Borderline-PatientInnen (Blum und Rahn 2014).

Das Basisprogramm von STEPPS gliedert sich in drei Schritte:

1. Die Krankheit erkennen und annehmen.

Vermittlung von objektiven Informationen über die

Erkrankung und der Abgleich mit dem subjektiven Erleben.

2. Der Umgang mit Emotionen.

Dazu werden fünf Fertigkeiten (distanzieren, kommunizieren, Gedanken hinterfragen, Ressourcen aktivieren, Probleme lösen) vermittelt.

3. Verhaltenstraining

In diesem Schritt geht es um den Transfer in verschiedene konkrete Lebensbereiche wie Ernährung, Bewegung, Freizeitgestaltung, Selbstfürsorge und zwischenmenschliche Beziehungen.

Das Gruppentraining ist in einer Art von Seminarform gestaltet in der die Eigeninitiative der GruppenteilnehmerInnen gefördert wird und die Rolle der „TrainerInnen“ sich hauptsächlich auf die Moderation der Gruppen, der Vermittlung von Wissen und die Strukturierung der Gruppensitzungen bezieht. Die Gruppensitzungen werden jeweils durch Entspannungs-, Achtsamkeits- oder Imaginationsübungen ergänzt.

Das STEPPS Programm ermöglicht eine langfristige Begleitung von Menschen mit BPD (Dauer bis zu zwei Jahren). Eine Fortführung als Selbsthilfegruppe ist möglich. Allerdings können aus dem Manual einzelne Einheiten herausgelöst und für gezielte kurzfristige Interventionen in anderen Settings genutzt werden.

\section{Welche besonderen Merkmale zeichnet das STEPPS Programm aus?}

Das STEPPS Programm zeichnet sich durch einige Besonderheiten aus, die es für den Einsatz in einer vernetzten ambulanten Versorgung von Menschen mit einer BPD geeignet erscheinen lassen. Wie in der DBT (Dialektisch-Behavioralen Therapie) bildet das
Verständnis der Borderline-Störung als emotionale Störung (emotionale Instabilität) die theoretische Basis. Nachdem im ersten Schritt ein Konsens zwischen den Betroffenen und den TrainerInnen $\mathrm{zu}$ diesem Modell hergestellt werden soll, werden die TeilnehmerInnen ermuntert, ihre sozialen Ressourcen bei der Bewältigung der emotionalen Instabilität und der Umsetzung ihrer persönlichen Ziele zu nutzen. $\mathrm{Zu}$ diesen sozialen Ressourcen gehören dabei nicht nur die professionellen HelferInnen, sondern auch Angehörige, Freunde und weitere soziale Bezugspersonen. Die Betroffenen sollen sich aktiv bei der Zusammenstellung des „Unterstützerteams“ bemühen. Dieser Trialogische Ansatz soll Unterstützung für den Transfer in den Alltag gewährleisten und schließt die Hilfen in Krisensituationen explizit ein. Die Betroffenen werden ermuntert, sich nicht nur auf eine Person zu konzentrieren. Ohnehin ist STEPPS als Ergänzung zu den bereits bestehenden Hilfen gedacht, etwa einer Einzeltherapie oder einer Anbindung an psychosoziale Hilfen und kann daher mit verschiedenen anderen Ansätzen bei der Bewältigung der Störung kombiniert werden (Silk 2008). Als Bindeglied dienen eine gemeinsame Sprache und das gemeinsame Verständnis der Erkrankung. In diesem Sinne lässt sich STEPPS in verschiedenen Versorgungskontexten nutzen, etwa als Ergänzung zu einer ambulanten Psychotherapie. Diese Öffnung des Programms im Hinblick auf psychosoziale Vernetzung ist dabei einer der wesentlichen strukturellen Unterschiede zur Vorgehensweise im Rahmen der DBT.

In den ersten Sitzungen des Verhaltenstrainings setzen sich die TeilnehmerInnen mit ihrer eigenen Situation auseinander, wobei auch die körperliche Gesundheit zum Thema wird, da die Erkrankung an einer BPD später mit verschiedensten schweren körperlichen Erkrankungen assoziiert ist (Keuroghlian et al. 2013). Auch der Konsum von Drogen und Alkohol steht im Fokus. Am Ende des Trainings werden zwischenmenschliche Themen in den Vordergrund gerückt: die Bitte um Hilfe, die Relativierung zwischenmenschlicher Mythen und die Pflege und der Aufbau sozialer Netzwerke wie auch partnerschaftlicher Beziehungen. Im folgenden STAIRWAYS Programm bilden dann zwischenmenschliche und psychosoziale Themen den Schwerpunkt (Tab. 2).

\section{Welche Anwendungsbereiche hat das STEPPS Programm?}

STEPPS dient vor allem der langfristigen ambulanten Versorgung von Menschen mit einer Borderline Erkrankung. Der aktive Umgang mit dem jeweiligen Kontext des betroffenen Menschen ist ein zentrales Ziel des Programms. Dazu ist aber jeweils eine Anpassung des Programms notwendig, was erfreulicherweise auch möglich ist. So gibt es beispielsweise Berichte, dass STEPPS erfolgreich in Gefängnissen, forensischen Kliniken, in der beruflichen Rehabilitation, mit 
betroffenen alleinerziehenden Müttern oder als Basis einer Selbsthilfegruppe genutzt wird und positive Effekte erzielt worden sind (Black et al. 2008, 2013).

Die Erfolge des STEPPS Programm verhalten sich auch relativ robust gegenüber Unterschieden im Schweregrad der Störung und Komorbidität bei den TeilnehmerInnen (Alesiani et al. 2014). Allerdings dürfen innerhalb der Gruppe die Unterschiede nicht zu groß sein, weil ansonsten die Abbruchquote steigt.

Bei einer Komorbidität mit affektiven Störungen (Depression, Angst etc.) scheint STEPPS einen positiven Effekt auf beide Störungen zu haben, etwa auf die Stimmung, die Lebensqualität oder das Ausmaß von Alkohol und Drogenmissbrauch (Riemann et al. 2014). Der Grad des Nutzens von der Teilnahme an dem STEPPS Programm ist abhängig, inwieweit das Programm komplett absolviert wird, was sich etwa an der Anzahl der Rehospitalisierungen, der Suizidversuche zeigen lässt (Alesiani et al. 2014). Auch Personen, die an einer Komplexen Persönlichkeitsstörung leiden (Komorbide Achse II Störung), scheinen von dem STEPPS Programme profitieren zu können (Black et al. 2016).

\section{Welche Rolle spielt das STEPPS Programm in der psychotherapeutischen und psychosozialen Versorgung von Borderline Kranken?}

Bei der Betrachtung der besonderen Merkmale des STEPPS Programms als langfristig angelegtes ambulantes Gruppenprogramm liegen die Stärken des Programms in der Strukturierung des Ablaufes, der Nutzung sozialer Unterstützung, der Vernetzung der Hilfen und der expliziten Thematisierung der psychosozialen Funktionsfähigkeit. Das Programm ist trialogisch angelegt und stellt damit sicher, dass die soziale Unterstützung und das soziale Netz der Betroffenen erhalten bleibt. In diesem Sinn wirkt STEPPS als Einstieg in eine indizierte Prävention. Außerdem wird die Selbsthilfe gefördert und dadurch auf Seiten der Helfer eine positive Haltung den Betroffenen gegenüber ermöglicht (Shanks et al. 2011). Durch den ergänzenden Charakter des STEPPS Trainings wird die Vernetzung gefördert und damit die Multiprofessionalität, weil nicht alle Elemente des STEPPS Trainings eine therapeutische Qualifikation im engeren Sinne erfordern. STEPPS enthält eine Möglichkeit zu Krisenintervention (vorwiegend durch Telefonkontakte) und senkt damit die Wahrscheinlichkeit von Notaufnahmen und nicht ambulant zu bewältigenden Krisen.

\section{Welche Vor- und Nachteile hat das STEPPS Programm im Vergleich mit anderen Hilfen für Borderline Kranken?}

STEPPS hat sich nach der Einführung mittlerweile in verschiedenen Regionen in Deutschland stabil etabliert, wozu die gute Anpassungsfähigkeit des Programms beigetragen hat. Als Hürde für die Vernetzung wirkt allerdings die nach Funktion und Kostenträger separierte Versorgungslandschaft, in der Abgrenzung vorherrscht. Daher sind multimodale Ansätze etwa in der ambulanten Psychotherapie wenig verbreitet. Ohnehin wird die ambulante Versorgung in den verschiedenen Segmenten von der Einzelbetreuung dominiert, zumal die Finanzierung systematischer Gruppenaktivitäten gegenwärtig schwierig ist. Der Ansatz, über eine gemeinsame Sprache zu einer besseren Zusammenarbeit zu kommen, stößt daher an Grenzen, was beispielsweise an der Verwendung des Begriffes „Therapie“ bei der Vernetzung der therapeutischen und psychosozialen Hilfen überaus deutlich wird. Die Anwendung von STEPPS stellt damit hierzulande keine Vernetzung her, kann aber eine Systematik für die Vernetzung bereitstellen, die für die Menschen mit einer BPD nicht verwirrend und widersprüchlich ist und die Verteilung von Aufgaben ermöglicht. Außerdem kann man die Betroffenen dabei einbinden, die vorhanden Möglichkeiten optimal zu nutzen. Die Anpassung an das gegenwärtige Versorgungssystem gelingt gegenwärtig allerdings aufgrund der beschriebenen Schwierigkeiten noch unvollständig.

Die Stärkung der Eigeninitiative ist ein besonders wertvoller Aspekt des STEPPS Trainings. So nehmen inzwischen eine große Zahl von Betroffenen an gemeinsamen Veranstaltungen teil und berichten von ihren Erfahrungen. Einige Gruppen werden von Betroffenen als Cotrainer begleitet. Mancherorts sind Selbsthilfegruppen entstanden, die das systematische Vorgehen des STEPPS Programms übernommen haben und sich damit strukturieren. Solche Entwicklungen sind aber nur in Regionen möglich, in denen die Vernetzung bereits funktioniert.

Im stationären Setting kann STEPPS allenfalls als Vorbereitung auf die ambulante Nachsorge eine Rolle spielen. Günstig ist, dass STEPPS sehr gut mit der DBT $\mathrm{zu}$ kombinieren ist, weil sich die theoretische Grundlage beider Verfahren entspricht. Im Unterschied zur DBT sind die in STEPPS vermittelten Fertigkeit wesentlich komplexer und bei extremen Stressreaktion oft nicht zugänglich, was die Überlegenheit der DBT beim Umgang mit Suizidalität und bei selbstverletzenden Verhalten erklärt. Nicht allen Betroffenen gelingt die Übersetzung der verwendeten Begrifflichkeiten, was die Erarbeitung einer gemeinsamen Sprache in der Behandlung von Borderline Kranken unterstreicht.

STEPPS ist sehr handlungsorientiert, fordert die Betroffenen aber noch erheblich im Hinblick auf Verständnis und Aufmerksamkeit. Mittlerweile gibt es eine Version in vereinfachter Sprache. Trotzdem stellen die Anforderungen für einige noch eine Hürde für die Teilnahme dar. Deswegen müssen in vielen Gruppen die Zeiten verkürzt, die Themen auf mehrere Termine aufgeteilt und damit das Programm zeitlich verlängert werden. 


\section{Welche Entwicklungen und Erweiterungen für das STEPPS Programm sind entwickelt oder möglich?}

Die Entwicklung von STEPPS ist dynamisch bei der Ausweitung von Themen und der Konzentration auf spezielle Zielgruppen. In der bisherigen Fassung fehlt das Thema Arbeit, obwohl dieses Thema bei der langfristigen Stabilisierung eine zentrale Rolle spielt. Dabei geht es um „tätig werden“ aber auch um „tätig bleiben“. Bei speziellen Zielgruppen sind bislang für Betroffene mit Adipositas und in Elternschaft eigene Gruppen gebildet worden. Es fehlt noch eine Variante für den Bereich der Betroffenen mit Substanzkonsum, ebenso eine Schulversion für die primäre Prävention.

\section{Abschluss}

Trotz vieler Fortschritte in der Behandlung und Versorgung von Menschen mit BPD gibt es noch viele offene Fragen, etwa wie eine langfristige Ausgrenzung der Klientel entgegengewirkt und eine gute soziale und berufliche Partizipation erreicht werden kann. Die zur Verfügung stehenden Behandlungsverfahren müssen in diesem Sinne weiterentwickelt und kombiniert werden. Das STEPPS Programm ist in vielerlei Hinsicht sicher ein wertvoller Baustein hin $\mathrm{zu}$ Entwicklung eines derartig übergreifenden Systems.

Interessenkonflikt E. Rahn gibt an, dass kein Interessenkonflikt besteht.

Open Access Dieser Artikel wird unter der Creative Commons Namensnennung 4.0 International Lizenz veröffentlicht, welche die Nutzung, Vervielfältigung, Bearbeitung, Verbreitung und Wiedergabe in jeglichem Medium und Format erlaubt, sofern Sie den/die ursprünglichen Autor(en) und die Quelle ordnungsgemäß nennen, einen Link zur Creative Commons Lizenz beifügen und angeben, ob Änderungen vorgenommen wurden.

Die in diesem Artikel enthaltenen Bilder und sonstiges Drittmaterial unterliegen ebenfalls der genannten Creative Commons Lizenz, sofern sich aus der Abbildungslegende nichts anderes ergibt. Sofern das betreffende Material nicht unter der genannten Creative Commons Lizenz steht und die betreffende Handlung nicht nach gesetzlichen Vorschriften erlaubt ist, ist für die oben aufgeführten Weiterverwendungen des Materials die Einwilligung des jeweiligen Rechteinhabers einzuholen.

Weitere Details zur Lizenz entnehmen Sie bitte der Lizenzinformation auf http://creativecommons.org/licenses/by/4. $0 /$ deed.de.

\section{Literatur}

Alesiani, R., Boccalon, S., Giarolli, L., Blum, N., \& Fossati, A. (2014). Systems Training for Emotional Predictability and Problem Solving (STEPPS): program efficacy and personality features as predictors of drop-out-An Italian study. Comprehensive Psychiatry, 55(4), 229-927.
Biskin, R.S. (2015). The lifetime course of borderline personality disorder. Canadian Journal of Psychiatry, 60(7), 303-308.

Black, D., Blum, N., Eichinger, L., McCormick, B., Allen, J., \& Sieleni, B. (2008). STEPPS: systems training for emotional predictability and problem solving in women offenders with borderline personality disorder in prison-a pilot study. CNS Spectrums, 13(10), 881-886.

Black, D. W., Blum, N., McCormick, B., \&Allen, J. (2013). Systems training for emotional predictability and problem solving (STEPPS) group treatment for offenders with borderline personality disorder. The Journal of Nervous and Mental Disease, 201(2), 124-129.

Black, D.W., Simsek-Duran, F., Blum, N., McCormick, B., \& Allen, J. (2016). Do people with borderline personality disorder complicated by antisocial personality disorder benefit from the STEPPS treatment program? Personal Ment Health, 10(3), 205-215.

Blum, N., \& John, S. D. (2008). STAIRWAYS - the next step in treatment for borderline personality disorder. Coralville: Level One Publishing. Blums Books

Blum, N.S., \& Rahn, E. (2014). Stepps: systems training for emotional predictability \& problem solving group treatment program for Borderline personality disorder. Köln: PsychiatrieVerlag.

Blum, N., St. John, D., Pfohl, B., Stuart, S., McCormick, B., Allen, J., et al. (2008). SystemsTraining for Emotional Predictability and Problem Solving (STEPPS) for outpatients with borderline personality disorder: a randomized controlled trial and 1-yearfollow-up. American Journal of Psychiatry, $165,468-478$.

Blum, N. S., Bartels, N. E., St. John, D., \&Pfohl, B. (2012). Systems training for emotional predictability and problem solving (STEPPS second edition): group treatment program for borderline personality disorder. Coralville: Level One Publishing. Blums Books

Bos, E. H., van Wel, E. B., Appelo, M.T., \& Verbraak, M. J.P.M. (2010). A randomized controlled trial of a Dutch version of systems trainingfor emotional predictability and problem solving for borderline personality disorder. The Journal of Nervous and Mental Disease, 198(4), 299-304.

Bos, E. H., van Wel, E. B., Appelo, M.T., \& Verbraak, M.J.P.M. (2011). Predictability and problem solving (STEPPS) for borderline personality problems in a 'real-world' sample: moderation by diagnosis or severity? Psychother Psychosom, 80, 173-181.

Botella Guillén, V., García-Palacios, A., Bolo Miñana, S., Baños, R., Botella, C., \& Marco, J.H. (2020). Exploring the effectiveness of dialectical behavior therapyversus systems training for emotional predictability and problem solving in a sample of patients with borderline. Journal of Personality Disorders.https://doi.org/10.1521/pedi_2020_34_477

Bozzatello, P., Bellino, S., Bosia, M., \& Rocca, P. (2019). Early detection and outcomein borderlinepersonalitydisorder. Frontiers in Psychiatry, 10, 1-16.

Frankenburg, F.R., \& Zanarini, M.C. (2005). The association between borderline personality disorder and chronic medical illnesses, poor health-related lifestyle choices, and costly forms of health care utilization. The Journal of clinical psychiatry, 65(12), 1660-1665.

Herpertz, S. C. (2020). ModularePsychotherapiebei Borderline Persönlichkeitsstörung. Psychotherapie, 65, 331-336.

Keuroghlian, A. S., Frankenburg, F. R., \& Zanarini, M. C. (2013). The relationship of chronic medicalillnesses, poor healthrelated lifestyle choices, and health care utilization to recovery status in borderline patients over a decade 
of prospective follow-up. Journal of Psychiatric Research, 47(10), 1499-1506.

Kramer, U., Temes, C.M., Magni, L.R., Fitzmaurice, G.M., Aguirre, B.A., Goodman, M., \& Zanarini, M.C. (2017). Psychosocial functioning in adolescents with and without borderline personality disorder. Personality and Mental Health, 11(3), 164-170.

Lis, S., \& Bohus, M. (2013). Social interaktion in borderline personality disorder. Current Psychiatrie Reports, 15, 338.

Riemann, G., Weisscher, N., Goossens, P. J. J., Draijer, N., Apenhorst-Hol, M., \& Kupka, R.W. (2014). The addition of STEPPS in the treatment of patients with bipolar disorder and comorbid borderline personality features: a protocol for a randomized controlled trial. BMC Psychiatry, 14, 172.

Schuppert, H.M., Giesen-Bloo, J., van Gemert, T.G., Wiersema, H.M., Minderaa, R.B., Emmelkamp, P.M.G., \& Nauta, M. H. (2009). Effectiveness of an emotion regulation group training for adolescents-a randomized controlled pilotstudy. Clinical Psychology and Psychotherapy, 16, 467-478.

Shanks, C., Pfohl, B., Blum, N., \& Black, D. W. (2011). Can negative attitudes toward patients with borderline personality disorder be changed? The effect of attending a STEPPS workshop. Journal of Personality Disorders, 25, 806-812.

Silk, K. R. (2008). Augmenting psychotherapy for borderline personality disorder: the STEPPS program. Am J Psychiatry, 165(4), 413-415.

Stone Michael, H. (2016). Long-term course of boderline personality disorder. Psychodynamic Psychiatry, 44, 449-474.
Wagner, T., Roepke, S., Marschall, P., Stiglmayr, C., Renneberg, B., Gieb, D., Dambacher, C., Matthies, S., Salbach-Andrae, H., Fleßa, S., \& Fydrich, T. (2013). Krankheitskosten der Borderline Persönlichkeitsstörung aus gesellschaftlicher Perspektive. Zeitschrift für Klinische Psychologie und Psychotherapie, 42(4), 242-255.

Van Wel, B., Bos, E. H., Appelo, M. T., Berendsen, E. M., Willgeroth, F. C., \&Verbraak, M. J. P. M. (2009). De effectiviteit van de vaardigheidstraining Emotieregulatiestoornis (VERS) in de behandeling van de Borderlinepersoonlijkheidsstoornis; een gerandomiseerd onderzoek. Tijdschrift voor psychiatrie, 51(5), 291-301.

Zanarini, M. C., Frankenburg, F., Hennen, J., Reich, D. B., \& Silk, K. (2006). Prediction of the 10-year course of borderline personality disorder. Am J Psychiatry, 163(5), 827-832.

Zanarini, M.C., Frankenburg, F. R., Reich, D. B., \& Fitzmaurice, G. (2010). The 10-year course of psychosocial functioning among patients with borderline personality disorder and axis II comparison subjects. Acta Psychiatrica Scandinavica, 122(2), 103-109.

Álvarez-Tomás, I., Ruiz, J., Guilera, G., \& Bados, A. (2018). Longterm clinical and functional course of borderline personality disorder: A meta-analysis of prospective studies. European Psychiatry, 56, 75-83.

Hinweis des Verlags Der Verlag bleibt in Hinblick auf geografische Zuordnungen und Gebietsbezeichnungen in veröffentlichten Karten und Institutsadressen neutral. 